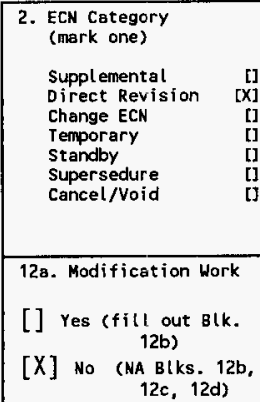

13a. Description of change
3. Originator's Name, Organization, MSIN, and Telephone No.
JR Bunch, SESC, L6-38, 376-2817
6. Project Title/No./Work Order No.
Flammable Gas Waste Tank Safety
9. Document Numbers Changed by this ECM (includes sheet no. and rev.)
EOF 610887 /WHC-SD-WM-ABU-002
cipisint Rev. 0

12b. Work Package Ho. $N / A$ 12c. Modification Work Complete

$N / A$

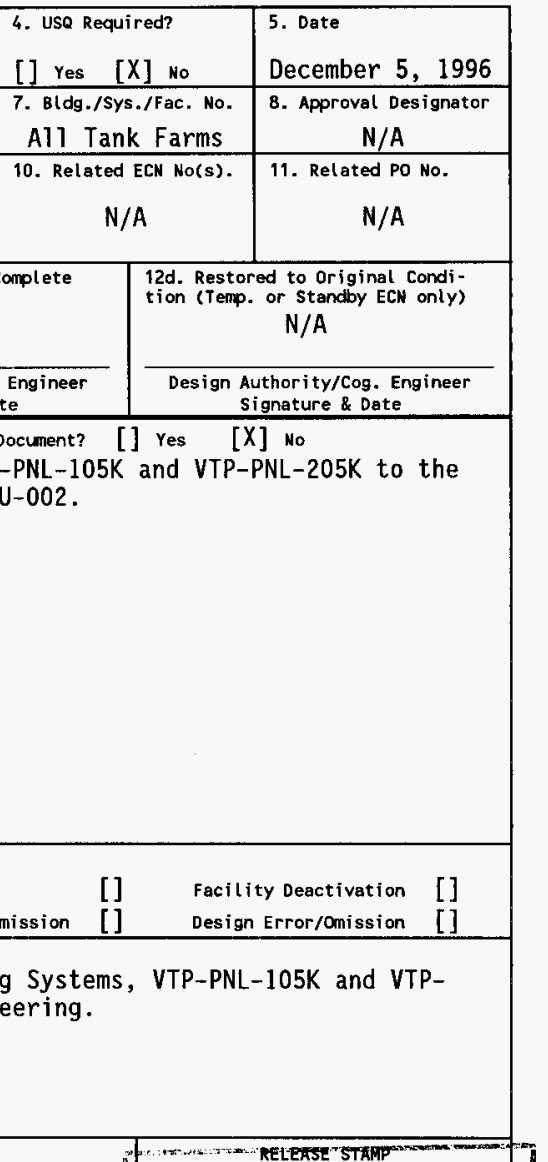

10. Related ECM No(s).

11. Related PO No.

N/A

$N / A$

Adding the Standard Hydrogen Monitoring Systems, VTP-PNL-105K and VTP-PNL-205K to the

Acceptance for Beneficial Use document, WHC-SD-WM-ABU-002.

(TBD-WM-038)

14a. Justification (mark one)

Criteria Charige $[X]$ Design Improvement

As-Found

[]

Facilitate const

[] Environmental

[] Const. Error/Omission

[]

Facility Deactivation Design Error/Omission []

14b. Justification Details

Formal turnover of the Standard D Hydrogen Monitoring Systems, VTP-PNL-105K and VTP-

PNL-205K to Operations, Maintenance, and Plant Engineering.

15. Distribution (include name, MSIN, and no. of copies)

RW Reed

S5-07 RL Legg

S5-06

MF Erhart

R1-5I CE Hanson

S7-12

GN Hanson

S5-05 JR Bunch

L6-38

WE Ross

S5-07 Project Files

S7-12

JE Geary

S5-07 TC Schneider

L6-37 


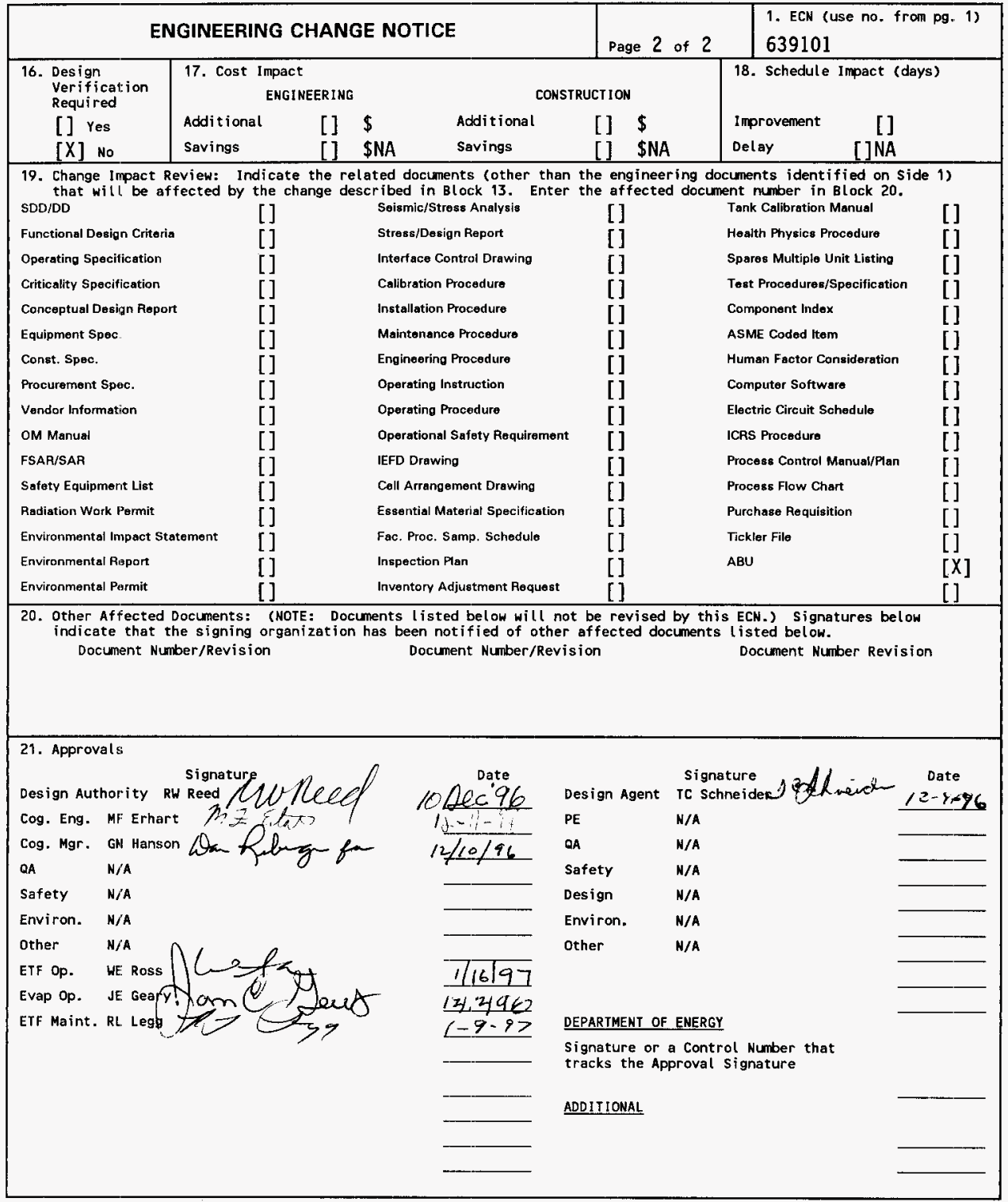




\title{
ACCEPTANCE FOR BENEFICIAL USE DOCUMENT
}

\author{
JR Bunch \\ SGN Eurisyg Servicea Corporation (SESC), Richland, WA 99352 \\ U.S. Department of Energy Contract DE-AC06-87RL10930 \\ EDT/ECN: 639101 \\ Org Code: 08E00 \\ BER Code: EW 3120072 \\ UC: 2030 \\ Charge Code: N2046 \\ Total Pages: $1 / 4 \mathrm{~km}$ \& $1 / 16 / 47$ \\ Key words: Standard Hydrogen Monitoring Syatems, sHMS, Stack Ammonia \\ Monitor, ABU, Acceptance for Beneficial Use. \\ Abstract: Adding the standard Hydrogen Monitoring Sygtems, VTP-PNL- \\ $105 \mathrm{~K}$ and VTP-PNL-205K to the Acceptance for Beneficial Use \\ document, WHC-SD-WM-ABU-002.
}

TRADEMARK DISCLAIMER. Reference herein to any specific commercial product, process, or service by trade name, trademark, manufacturer, or otherwise, does not necessarily constitute or imply its endorsement, recomendation, or favoring by the United States Goverruent or any agency thereof or its contractors or subcontractors.

Printed in the United States of America. To obtain copies of this document, contact: HHC/BCS Document Control Services, P.0. BoX 1970, Mailstop H6-08, Richtand WA 99352, Phone (509) 372-2420; Fax (509) 376-4989.
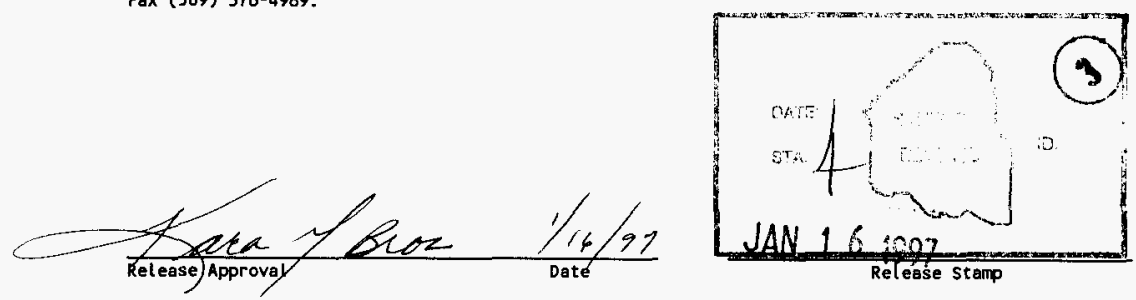

\section{Approved for Public Release}


(2) ritle

STANDARD HYDROGEN MONITORING SYSTEMS ACCEPTANCE FOR BENEFICIAL USE

CHANGE CONTROL RECORD

\begin{tabular}{|l|l|} 
(3) Revision & (4) Description of Change - Replace, Add, and Delete Pages \\
\hline 0 & (7) EDT 610887 \\
\hline 1 RS & $\begin{array}{l}\text { Adding the Standard D Hydrogen Monitoring } \\
\text { Syatemg, VTP-PNL-105K and VTP-PNL-205K, to } \\
\text { the WHC-SD-WM-ABU-002 document. ECN } \\
639101\end{array}$ \\
\hline
\end{tabular}

\begin{tabular}{|c|c|}
\hline (5) Cog. Engr. & (6) Cog. Mgr. \\
\hline $\begin{array}{l}\text { JR Bunch } \\
7 / 2 B u n c h \\
12 / 9 / 96\end{array}$ & $\begin{array}{l}\text { CE Hanson } \\
\text { Cophlfors }\end{array}$ \\
\hline
\end{tabular}

\begin{tabular}{|l|l|}
\hline & 639101 \\
\hline &
\end{tabular}


TABLE OF CONTENTS

INDEX OF ABU DOCUMENTATION - STANDARD HYDROGEN MONITORING SYSTEMS * 2

ABU INDEX - STANDARD HYDROGEN MONITORING SYSTEMS • . . . . . . . . 3

DOCUMENTATION REQUIRED for ACCEPTANCE FOR BENEFICIAL USE * • • . . 7

TANK FARM DRAWINGS RELATED TO STANDARD HYDROGEN MONITORING SYSTEM CONSTRUCTION . . . . . . . . . . . . . . . . . . . . . . . . . . . . 9

STANDARD HYDROGEN MONITORING SYSTEM INSTALLATION WORK PACKAGES • • 13 


\section{INDEX OF ABU DOCUNENTATION - STANDARD HYDROGEN MONITORING SYSTEMS}

This index is intended to serve two purposes:

1) it is a "roadmap" to be used by Waste Tank Operations personnel of all the required turnover documentation for the standard Hydrogen Monitoring systems,

2) it is also intended to demonstrate formal completion of all requirements of the approved $A B U$ checklist for this project.

The following table lists each item required by the approved standard Hydrogen Monitoring syatem ABU checklist and identifies the corresponding document control number. An explanation of any exceptions or open items is included in the table. Copies of the original ABU checklists are attached as a convenient reference.

TANK

A- 101

AN- 103

AN-104

AN -105

241-AN STACK

AW-101

241-AW STACK

$\mathrm{AX}-101$

$\mathrm{Ax}-103$

s-102

s-111

S-112

sX-101

sX-102

sX -103

sx-104

sx-105

$\mathrm{sx}-106$

sX-109

SY-101

SY-101

SY-101

SY-103

$\mathrm{T}-110$

U-103

U-105

U-107

$\mathrm{U}-108$

$\mathrm{U}-109$

\section{CABINET}

VTP-PNL-805A

VTP-PNL-105C

VTP-PNL-105D (temporary gas chromatograph not included)

VTP-PNL-105E

VTP-PNL-105K (temporary ammonia monitor not included)

VTP-PNL-205A

VTP-PNL-205K (temporary ammonia monitor not included)

VTP-PNL-905A

VTP-PNL-905C

VTP-PNL-3305B

VTP-PNL-3305K

VTP-PNL-3305L

VTP-PNL-3405A

VTP-PNL-3405B

VTP-PNL-3405C

VTP-PNL-3405D

VTP-PNL-3405E

VTP-PNL-3405F

VTP-PNL-3405I

NAN-101-J

NAN $-101-\mathrm{K}$

NAN-101-L

NAN-103-J

VTP-PNL-3705J

VTP-PNL-3805C

VTP-PNL-3805E

VTP-PNL-3805G

VTP-PNL-3805H

VTP-PNL-3805 I 


\begin{tabular}{|c|c|c|}
\hline \multicolumn{3}{|c|}{ ABU INDEX - STANDARD HYDROGEN MONITORING SYSTEMS } \\
\hline Checklist Item & Document Number and Title & Comments \\
\hline ABU Checklist & $\begin{array}{l}\text { EDT file \#609616 } \\
\text { EDT file \#614197 } \\
\end{array}$ & Copy at tached \\
\hline Safety Assessment & 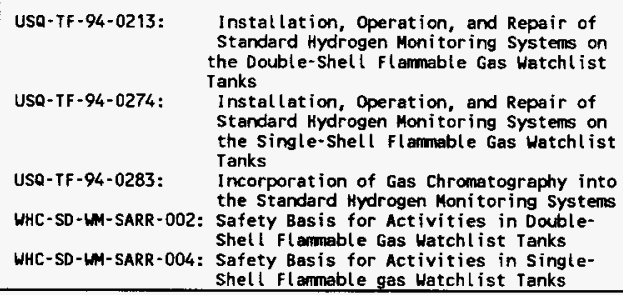 & \\
\hline Safety Equipment List & $\begin{array}{l}\text { WHC-SD-WM-SEL-026: Double Shell Tanks Inter im Safety } \\
\text { Equipment List } \\
\text { WHC-SD-WH-SEL-027: } \\
\text { Single Shell Tanks Inter im Safety } \\
\text { Equipment List }\end{array}$ & $\begin{array}{l}\text { SEL's } 026 \text { and } 027 \text { are presently in review } \\
\text { for approval. } \\
\text { (TBD-LM-038) }\end{array}$ \\
\hline Engineering Task Plan & $\begin{aligned} & \text { WHC-SD-LM-UP-251: } 200 \text { Area Watchlist Waste Tank Standard } \\
& \text { Hydrogen Monitoring System Installation } \\
& \text { Engineering Work Plan }\end{aligned}$ & \\
\hline Functional Design Criteria & 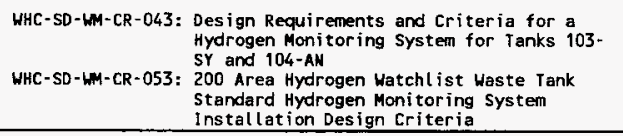 & \\
\hline System Design Description & 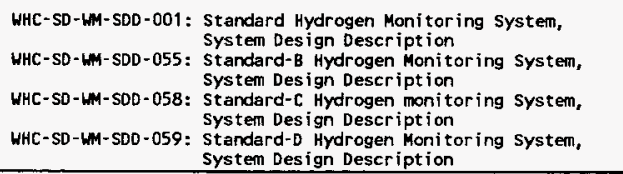 & \\
\hline
\end{tabular}




\begin{tabular}{|c|c|c|}
\hline \multicolumn{3}{|c|}{ ABU INDEX - STANDARD HYDROGEN MONITORING SYSTEMS } \\
\hline Acceptance Test Procedures & 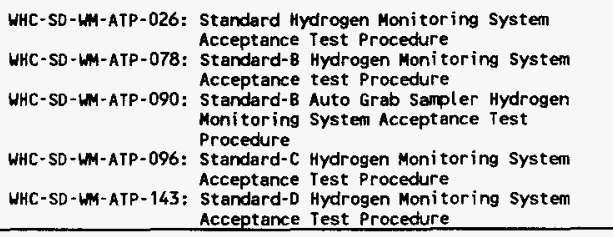 & \\
\hline Acceptance Test Reports & $\begin{array}{l}\text { WHC-SD-WM-ATR-026: } \begin{array}{l}\text { Standard Hydrogen Monitoring System } \\
\text { Acceptance Test Report }\end{array} \\
\text { WHC-SD-WM-ATR-078: } \\
\text { Standard-B Hydrogen Monitoring System } \\
\text { Acceptance Test Report }\end{array}$ & \\
\hline Operating Procedure & $\begin{array}{l}\text { Tank Farm Plant Operating Procedure ro-040-040: Operate the } \\
\text { Standard B or C Hydrogen Monitoring Systems } \\
\text { Tank Farm PLant Operating Procedure T0-040-041: Operate the } \\
\text { Standard D Hydrogen Monitoring Systems }\end{array}$ & \\
\hline Surveillance Procedure & $\begin{array}{l}\text { Tank Farm Plant Operating Procedure 10-040-040: Operate the } \\
\text { Standard B or C Hydrogen Monitoring Systems } \\
\text { Tank Farm Plant Operating Procedure TO-040-041: Operate the } \\
\text { Standard D Hydrogen Monitoring Systems }\end{array}$ & \\
\hline Calibration Procedure & $\begin{array}{ll}\text { 6-TF-408: } & \text { Whittaker Model 1140038-10 Hydrogen Cell with } \\
\text { Newport Infinity electronics, Calibrations } \\
\text { W-TF-440: }\end{array}$ & \\
\hline Preventative Maintenance Procedures & JCS PM/S Component Recall system & $\begin{array}{l}\text { All SHMS equipment requiring preventive } \\
\text { ma intenance and referenced maintenance } \\
\text { procedures, have been placed into the JCS } \\
\text { PM/S Component Recall system }\end{array}$ \\
\hline
\end{tabular}




\section{ABU INDEX - STANDARD HYDROGEN MONITORING SYSTEMS}

\section{PM/S Data Sheets}

perating and Maintenance Manual

Equipment Specifications

Procurement Specifications

Construction Specifications

Installation Drawings

As-built Drawings

EFD Drawings

Drawing Tree

Incorporate outstanding project generated ECNs

Sof tware Configuration Managenent Plan
JCS PM/S Component Recall System

WHC-WM-OMM-005: Standard Hydrogen Moni toring System

Operation and Maintenance Manual

Standard-B Hydrogen Moni toring System

WHC-WM-OMM-018: Operation and Maintenance Manual

Operation and Maintenance Manual

WHC-LM-OMM-020: Standard D Hydrogen Monitoring System

Operation and Maintenance Manual

WHC-SO-WM-EII-003: Standard Hydrogen Monitoring System Equipment Installation Instructions

WHC-SD-WM-EII-003: standard Hydrogen Monitoring System Equipment Instal lation Instructions

WHC-SD-W-EII-003; Standard Hydrogen Monitoring Systen Equipment Installation Instructions

See drawing H-2-87275: Standard hydrogen Monitoring System Drawing Index Tree, WHC-SD-WM-EII-003: Standard Hydrogen Monitoring System Equipment Installation Instructions.

W-369 Project ECN's $W-369-1$ through $w-369-44,46,48,49$,

$50,51,53,54$, and 66 , and the attached Tank Farm Drawings

Related To Standard hydrogen Monitoring System Construction

list for complete listing of installation drawings.

See drawing H-2-87275: Standard Hydrogen Monitoring Systen Drawing Index Tree, WHC-SD-WM-EII-003: Standard Hydrogen Monitoring System Equipment Installation Instructions, and

the attached Tank Farm Drawings Related To Standard Hydrogen

Monitoring System Construction list for listing of as-built drawings.

See the attached Tank Farm Drawings Related To Standard Hydrogen Monitoring System Construction list.

H-2-87275: Standard Hydrogen Monitoring System Drawing Index Tree

\section{Completed}

WHC-SD-LM-CSCM-013: Standard Hydrogen Monitoring System Management $\mathrm{Pl}$ an
The Operation and Maintenance Manuls List equipment in the SHMS that require

calibration, including their range and

accuracy. All sHas equipment requiring

calibration has been placed into the JCS

PM/S Component Recall System. 


\section{ABU INDEX - STANDARD HYDROGEN MONITORING SYSTEMS}

\begin{tabular}{|c|c|c|}
\hline Software Design Description & $\begin{aligned} & \text { WHC-SD-LM-CSOD-004: } \text { Standard Hydrogen Moni tor ing System } \\
& \text { Computer Software Design Descript ion }\end{aligned}$ & \\
\hline Training Plan & $\begin{array}{l}\text { Course No. 351405: Tank farms Standard Hydrogen Monitoring } \\
\text { Systems } \\
\text { Course No. 356006: Standard Hydrogen Monitoring Systems } \\
\text { Maintenance }\end{array}$ & $\begin{array}{l}\text { A training plan was not written and is not } \\
\text { needed now, since the training courses are } \\
\text { in place. }\end{array}$ \\
\hline Training to Operating Crews & $\begin{array}{c}\text { Course no. 351405: Tank Farms Standard Hydrogen Monitoring } \\
\text { Systems }\end{array}$ & \\
\hline Training to Maintenance Crews & Course no. 356006: Standard Hydrogen Monitoring Systems & \\
\hline Training Mock-up & Presently located in the 306 building & \\
\hline Inspection Plan & $\begin{array}{r}\text { WHC-SD-W369-QAPP-001: W-369 Project Specific Quality } \\
\text { Assurance Plan, Table 10-2 }\end{array}$ & \\
\hline OAPP & $\begin{array}{ll}\text { WHC-SD-W369-QAPP-001: W-369 Project Specific Quality } \\
\text { Assurance Plan } \\
\text { WHC-SD-WM-OAPP-018: } \\
\text { Tank Waste Projects Quality Assurance } \\
\text { Program Plan }\end{array}$ & \\
\hline Vender Information files & $\begin{array}{l}\text { VI22129: Vendor File Information for Gas Monitoring Systems } \\
\text { VI22192: Vendor File Information for the Standard Hydrogen } \\
\text { Monitoring Systems }\end{array}$ & \\
\hline Spare Parts List & $\begin{array}{ll}\text { Whittaker Cell } & \# 1090020-2 \\
\text { Newport Monitor } & \# \text { \#NFU-0-0-1-0-DCi } \\
\text { Omega Controller } & \# C N 9121 \mathrm{~A} \\
\text { Omega Indicator } & \# C N 111 \mathrm{~A} \\
\text { Matheson Filter } & \# 6134-\mathrm{TBFF} \\
\text { Generant Filter } & \# 25500 \\
\text { Senior Flextronics/Metal Bellows Sample } & \\
\text { Pump Assembly } & \# 29678 M 1 \\
\end{array}$ & - \\
\hline Spare Parts in Stock & Spare parts are located in the $2101 \mathrm{M}$ warehouse. & \\
\hline SHMS Projects Punchlists (open Items) & Project $\mathrm{H}-369$ & No open $w-369$ Project punchl ist items. \\
\hline Installation Hork Plan & $\begin{array}{l}\text { See the attached Standard Hydrogen Monitoring System } \\
\text { Installation Work Package list. }\end{array}$ & $\begin{array}{l}\text { The Standard Hydrogen Monitoring Systems } \\
\text { were installed per the JCS Work Package } \\
\text { System. }\end{array}$ \\
\hline
\end{tabular}




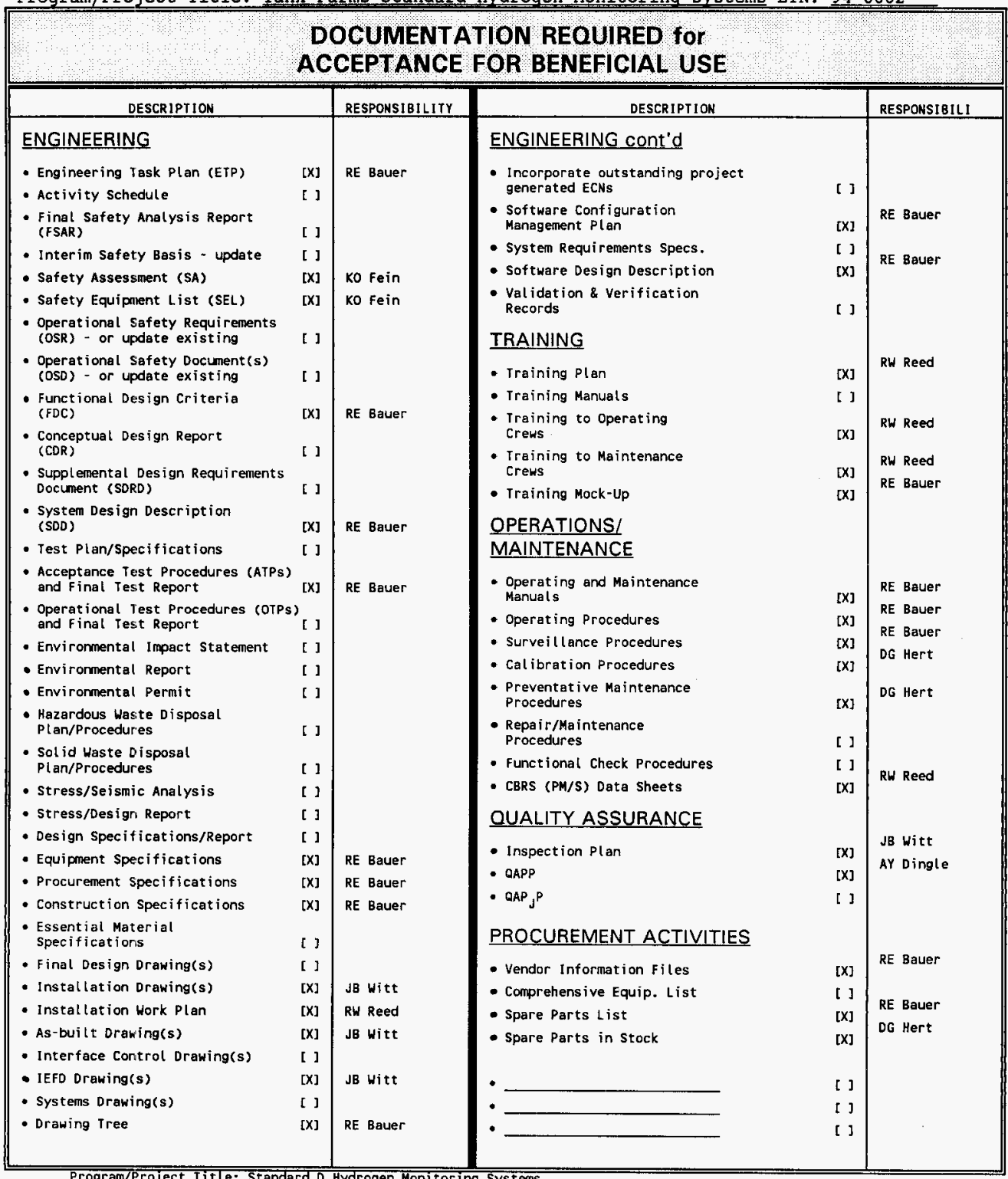




\section{DOCUMENTATION REQUIRED for ACCEPTANCE FOR BENEFICIAL USE}

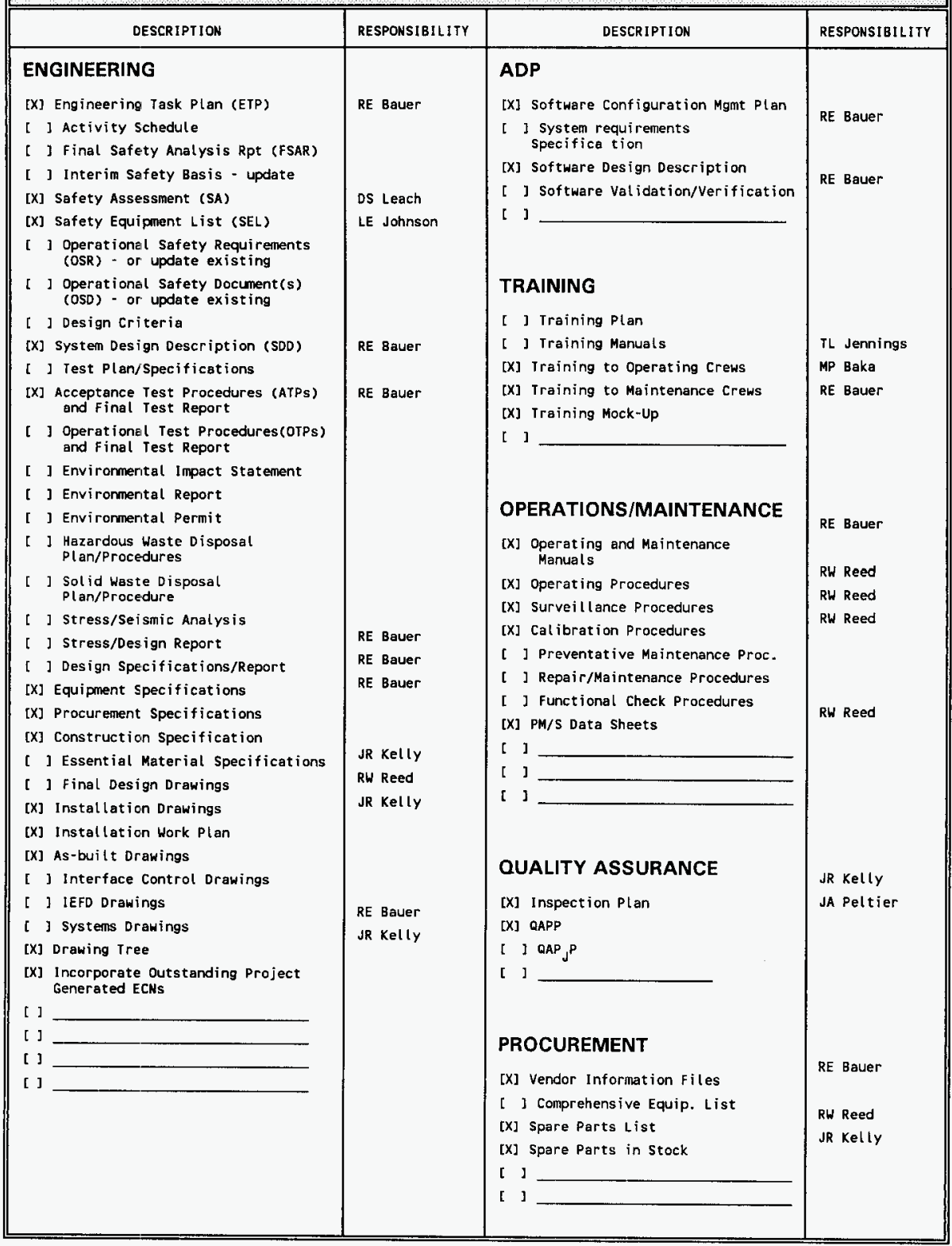


TANK FARM DRAKINGS RELATED TO STANDARD HYDROGEN MONITORING SYSTEM CONSTRUCTION

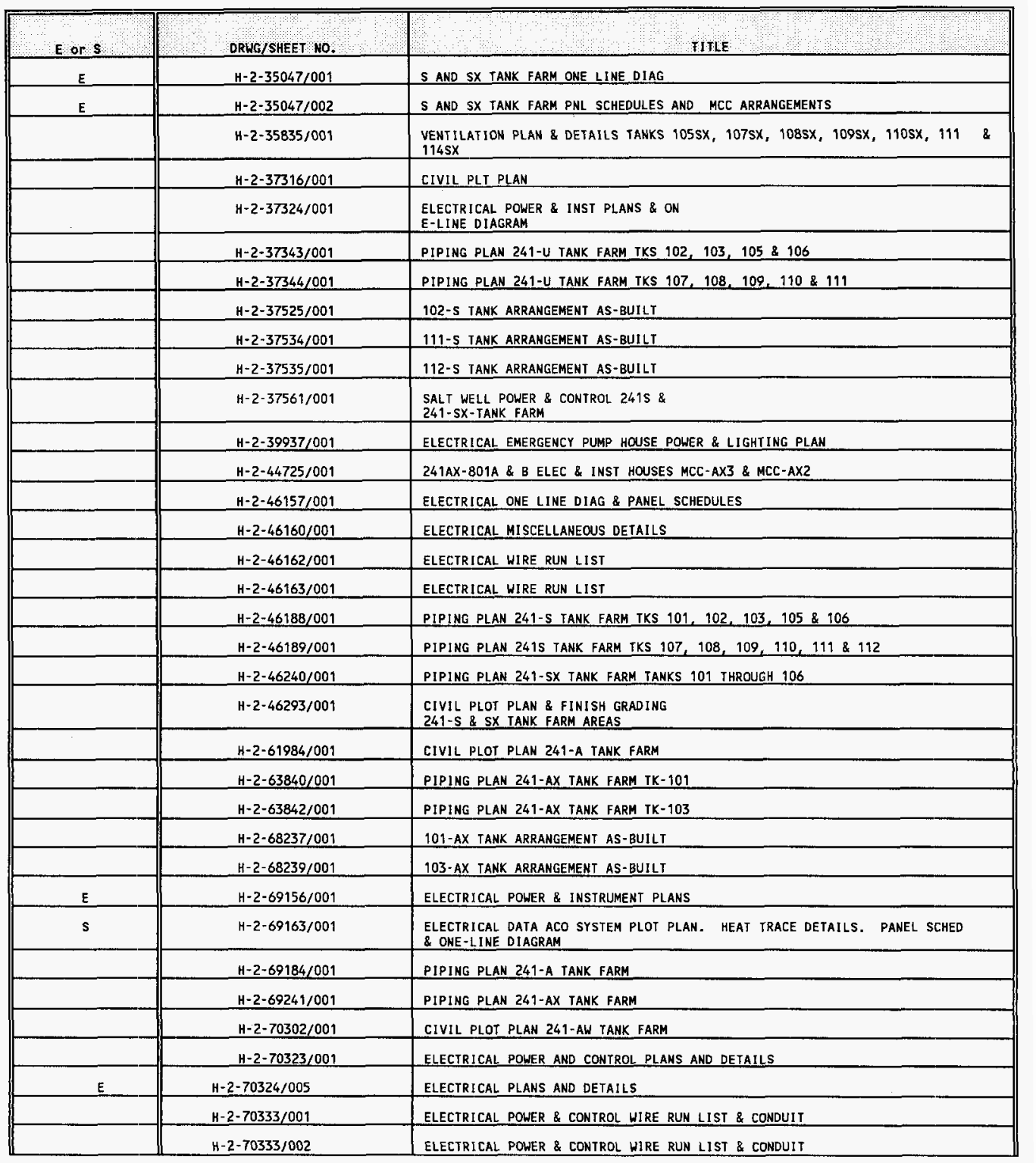




\begin{tabular}{|c|c|c|}
\hline & $\mathrm{H}-2-70333 / 003$ & ELECTR ICAL POWER \& CONIROL WIRE RUN LIST \& CONDUIT \\
\hline & $\mathrm{H}-2-70333 / 004$ & ELECTRICAL INSTRUMENT WIRE RUN LIST AND CONDUIT SCHEDULE \\
\hline & $\mathrm{H}-2-70333 / 005$ & ELECTRICAL INSTRUMENT WIRE RUN LIST AND CONDUIT SCHEDULE \\
\hline & $\mathrm{H}-2-70333 / 008$ & ELECTRICAL CONDUIT WIRE RUN SCHEDULE \\
\hline & $\mathrm{H}-2-70337 / 001$ & HVAC/PIPING VENT PIPING PLAN 1 241-AW TANK FARM \\
\hline & $\mathrm{H}-2-70341 / 001$ & HVAC/PIPING STANDARD TANK FARM DETAILS \\
\hline & $\mathrm{H}-2-71901 / 001$ & CIVIL SITE PLAN 241-AN TANK FARM \\
\hline$s$ & $\mathrm{H}-2-71925 / 001$ & ELECTRICAL POWER AND CONTROL PLANS \& DETAILS \\
\hline $\mathbf{s}$ & $\mathrm{H}-2-71925 / 002$ & ELECTRICAL POWER \& CONTROL PLAN \& DETAILS \\
\hline s & H-2-71925/003 & ELECTRICAL POWER AND CONTROL PLANS AND DETAILS \\
\hline $\mathbf{s}$ & $H-2-71925 / 004$ & ELECTRICAL POWER \& CONTROL PLANS \& DEIAILS \\
\hline \multirow[t]{6}{*}{$s$} & $H-2-71925 / 005$ & ELECTRICAL POWER \& CONTROL PLANS \& DETAILS \\
\hline & $H-2-71934 / 001$ & ELECTRICAL POWER \& CONTROL WIRE RUN LISI \\
\hline & $H-2-71934 / 002$ & ELECTRICAL POWER \& CONIROL WIRE RUN LIST \\
\hline & $\mathrm{H}-2-71934 / 003$ & ELECTRICAL POWER \& CONTROL WIRE RUN LIST \\
\hline & $H-2-71934 / 005$ & $\begin{array}{l}\text { ELECTRICAL INSIRUMENT WIRE RUN LIST } \\
\text { \& CONDUIT SCHEDULE }\end{array}$ \\
\hline & $\mathrm{H} \cdot 2 \cdot 71935 / 002$ & HVAC FLOW \& CONTROL DIAGRAM \\
\hline$s$ & $\mathrm{H}-2-71936 / 001$ & HVAC EQUIPMENT PLAN ELEVATIONS \& DETAILS \\
\hline $\mathbf{s}$ & $\mathrm{H}-2-71936 / 002$ & HVAC EQUIPMENI PLAN ELEVATIONS \& DETAILS \\
\hline $\mathrm{s}$ & $\mathrm{H}-2-71992 / 001$ & PIPING PLAN TANK 102 \\
\hline$\$$ & $\mathrm{H}-2-71994 / 001$ & PIPING PLAN TANK 104 \\
\hline \multirow[t]{14}{*}{$\underline{s}$} & $\mathrm{H}-2-71995 / 002$ & PIPING PLAN TANK 105 \\
\hline & $\mathrm{H}-2-72031 / 001$ & PIPING MISC DETAILS \\
\hline & $\mathrm{H}-2-73064 / 001$ & $\begin{array}{l}\text { PIPING WASTE TANK ISOLATION } \\
241-T-110\end{array}$ \\
\hline & $H-2-73151 / 001$ & $\begin{array}{l}\text { PIPING WASTE TANK ISOLATION } \\
241-\mathrm{U}-103\end{array}$ \\
\hline & $H-2-73153 / 001$ & $\begin{array}{l}\text { PIPING WASTE TANK ISOLATION } \\
241-\mathrm{U}-105\end{array}$ \\
\hline & $H-2-73155 / 001$ & $\begin{array}{l}\text { PIPING WASTE TANK ISOLATION } \\
241-U-107\end{array}$ \\
\hline & $H-2-73156 / 001$ & $\begin{array}{l}\text { PIPING WASTE TANK ISOLATION } \\
241-U-108\end{array}$ \\
\hline & $H-2-73157 / 001$ & $\begin{array}{l}\text { PIPING WASTE TANK ISOLATION } \\
241-\mathrm{U}-109\end{array}$ \\
\hline & $H-2 \cdot 73182 / 001$ & $\begin{array}{l}\text { PIPING WASTE TANK ISOLATION } \\
\text { TK } 241-5-102\end{array}$ \\
\hline & H-2-73190/001 & $\begin{array}{l}\text { PIPING WASTE TANK ISOLATION TK } \\
24 i-S-11 \uparrow\end{array}$ \\
\hline & H-2-73191/001 & $\begin{array}{l}\text { PIPING WASTE TANK ISOLATION } \\
241-5-112\end{array}$ \\
\hline & $H-2-73218 / 001$ & $\begin{array}{l}\text { PIPING WASTE TANK ISOLATION } \\
241-5 X-101\end{array}$ \\
\hline & $H-2-73219 / 001$ & $\begin{array}{l}\text { PIPING WASTE TANK ISOLATION } \\
241-5 \mathrm{X}-102\end{array}$ \\
\hline & $H-2-73220 / 009$ & $\begin{array}{l}\text { PIPING WASTE TANK ISOLATION } \\
249-5 X-103\end{array}$ \\
\hline
\end{tabular}




\begin{tabular}{|c|c|c|}
\hline & $H \cdot 2-73221 / 001$ & $\begin{array}{l}\text { PIPING WASTE TANK ISOLATION } \\
241-\mathrm{S} X-104\end{array}$ \\
\hline & H-2-73222/001 & $\begin{array}{l}\text { PIPING WASTE TANK ISOLATION } \\
241-\mathrm{S} \times-105\end{array}$ \\
\hline & $H-2-73223 / 001$ & $\begin{array}{l}\text { PIPING WASTE TANK ISOLATION } \\
241-\mathrm{SX} \cdot 106\end{array}$ \\
\hline & $H-2-73376 / 001$ & $\begin{array}{l}\text { PIPING WASTE TANK ISOLATION } \\
241-A X-101\end{array}$ \\
\hline & $H-2-73380 / 001$ & $\begin{array}{l}\text { PIPING WASTE TANK ISOLATION } \\
241-A X-103\end{array}$ \\
\hline & $H-2-73388 / 001$ & $\begin{array}{l}\text { PIPING WASTE TANK ISOLATION } \\
241-\mathrm{A}-101\end{array}$ \\
\hline & $H-2-73682 / 001$ & $\begin{array}{l}\text { ELECIRICAL JET PUIMP CONTROL ON } \\
\text { E-LINE ANO ELEMENTARY DIAGRAM }\end{array}$ \\
\hline \multirow[t]{8}{*}{ E } & $H-2-73682 / 002$ & $\begin{array}{l}\text { ELECIRICAL JET PUMP CONTROL ON } \\
\text { E-LINE ANO ELEMENTARY DIAGRAM }\end{array}$ \\
\hline & $H-2-73692 / 001$ & ELECIRICAL/INSTRUMENTATION WIRE RUN LIST \\
\hline & $H-2-73692 / 002$ & ELECTRICAL/INSTRUMENTATION WIRE RUN LISI \\
\hline & $\mathrm{H}-2-73956 / 001$ & ELECTRICAL/INSTRUMENTATION WIRE RUN LIST \\
\hline & $\mathrm{H}-2-73958 / 001$ & ELECTRICAL DISTRIBUTION PLAN 241-T TANK FARM \\
\hline & $\mathrm{H}-2-73978 / 001$ & PIPING \& INSIRUMENTAIION PLAN 241-T TANK FARM \\
\hline & $\mathrm{H}-2-77723 / 001$ & PIPING/ELEC PLAN 241-AW TANK FARM \\
\hline & $\mathrm{H}-2-85301 / 004$ & PANELBOARD SCHEDULE \\
\hline $\mathbf{E}$ & $\mathrm{H}-2-85303 / 001$ & PANELBOARD SCHEDULE INDEX \\
\hline $\mathbf{E}$ & $H+2-85303 / 004$ & PANELBOARD SCHEDULE \\
\hline E & $\mathrm{H}-2-90905 / 001$ & DWG LIST/HVAC FLOW CONIROL DIAGRAM \& NOTES \\
\hline \multirow[t]{7}{*}{$s$} & $H-2-90906 / 001$ & HVAC-K1 SYSIEM EQUIPMENI PLAN \& ELEVATIONS \\
\hline & $\mathrm{H}-2-90930 / 001$ & ELECTRICAL PLANS \& DETAILS \\
\hline & $\mathrm{H}-2-91111 / 001$ & ELECIRICAL WIRE RUN LIST AND CONDUIT SCHEDULE \\
\hline & $\mathrm{H}-2-91111 / 002$ & ELECTRICAL WIRE RUN LIST AND CONDUIT \\
\hline & $\mathrm{H}-2-91122 / 001$ & ELEC/INSTM WIRE RUN LIST 241-T TANK FARM \\
\hline & $\mathrm{H}-2-93664 / 001$ & ELECTRICAL HEAT TRACE \& INSTR BLDG PLANS \\
\hline & $\mathrm{H}-2-93668 / 001$ & ELECTRICAL WIRE RUN LIST \\
\hline \multirow[t]{13}{*}{ E } & $\mathrm{H}-2-99389 / 002$ & ONE LINE DIAGRAMS \\
\hline & $\mathrm{H}-2-815299 / 002$ & I-TANK FARM CONDUIT PLAN \\
\hline & $H-2-815438 / 006$ & TMACS T-TANK FARM WIRE RUN LIST \\
\hline & $\mathrm{H}-2-820778 / 001$ & IMACS S-TANK FARM INSTM. PLAN \\
\hline & $\mathrm{H}-2-820778 / 002$ & TMACS S-TANK FARM CONDUIT PLAN \\
\hline & $\mathrm{H}-2-820778 / 005$ & TMACS S-TANK FARM WIRE RUN LIST \\
\hline & $\mathrm{H}-2-820784 / 001$ & TMACS SX-TANK FARM INSTM. PLAN \\
\hline & $\mathrm{H}-2-820784 / 002$ & TMACS SX-TANK FARM CONDUIT PLAN \\
\hline & $H-2-820804 / 001$ & TMACS U-TANK FARM INSTM. PLAN \\
\hline & $H-2-820804 / 002$ & TMACS U-TANK FARM CONDUIT PLAN \\
\hline & H-2-820804/005 & TMACS U-TANK FARM WIRE RUM LIST \\
\hline & $\mathrm{H}-2-920804 / 006$ & TMACS U-TANK FARM WIRE RUN LIST \\
\hline & $H-2-140310 / 002$ & TMACS AN-TANK FARM INSTM PLAN VIEW \\
\hline
\end{tabular}


WHC-SD-WM-ABU-002, Rev . 1

\begin{tabular}{|c|c|c|}
\hline & $H-2-140310 / 007$ & TMACS AN-TANK FARM WIRE RUN LIST \\
\hline & $\mathrm{H}-2-820792 / 002$ & TMACS AW-TANK FARM INSTM PLAN \\
\hline E & $\mathrm{H}-14-20101 / 002$ & VENTILATION TANK PRIMARY SYSTEM VTP O\&M SYSTEM PRID \\
\hline $\mathbf{E}$ & $H-14-20102 / 002$ & VENTILATION TANK PRIMARY SYSTEM VTP O\&M SYSTEM P\&ID \\
\hline E & $H-14-30001 / 002$ & ELECTRICAL ONE LINE DIAGRAM EDS-MCC-102 \\
\hline E & $H-14-30001 / 008$ & PANELBOARD SCHEDULE \\
\hline$E$ & $\mathrm{H}-14-30001 / 019$ & PANELBOARD SCHEDULE \\
\hline E & $\mathrm{H}-14-30002 / 002$ & ELECTRICAL EDS ONE LINE DIAGRAM \\
\hline E & $H-14-30002 / 007$ & PANELBOARD SCHEDULE \\
\hline
\end{tabular}




\section{STANDARD HYDROGEN MONITORING SYSTEM INSTALLATION WORK PACKAGES}

\begin{tabular}{|c|c|}
\hline WORK PACKAGE HUMBER & TITLE \\
\hline $2 E-94-00750 / W$ & \%241-A INSPECT FOR OBSTRUCTIONS AT 101-A \\
\hline $2 \mathrm{E}-94-00751 / \mathrm{W}$ & \%241-AX INSPECT FOR OBSTRUCTIONS AT 101-AX \\
\hline $2 \mathrm{E}-94-00752 / \mathrm{W}$ & $\% 241$-AX INSPECT FOR OBSTRUCIIONS AT 103-AX \\
\hline $2 E-94-00579 / W$ & $\% 241$-AW INSTALL 101 -AW SHMS \\
\hline $2 \mathrm{E}-94-00580 / \mathrm{W}$ & $\approx 241$-AN INSTALL 103-AN SHMS \\
\hline $2 E-94-00581 / \mathrm{W}$ & $\% 241$-AN INSTALL 104 -AN SHMS \\
\hline $2 E-94-00582 / W$ & $\% 241$-AN INSIALL 105 -AN SHMS \\
\hline $2 E-94-01008 / M$ & \%241-A INSTALL 101-A SHMS \\
\hline $2 E-94-01009 / M$ & $\% 241$-AX INSTALL 101 -AX SHMS \\
\hline $2 E-94-01010 / M$ & $\% 241$-AX INSTALL 103 -AX SHMS \\
\hline $2 \mathrm{~W}-94-00908 / \mathrm{K}$ & 241-SX INSTALL 101-SX VAPOR PROBE \\
\hline $2 \mathrm{H}-94-00909 / \mathrm{K}$ & 241-SX INSTALL 102-SX VAPOR PROBE \\
\hline $2 \mathrm{~W}-94-00910 / \mathrm{K}$ & 241 -SX INSTALL 103-SX VAPOR PROBE \\
\hline $2 W-94-00911 / K$ & 241-SX INSTALL 104-SX VAPOR PROBE \\
\hline $2 W-94-00912 / K$ & $241-5 X$ INSTALL $105-5 X$ VAPOR PROBE \\
\hline $2 \mathrm{~W}-94-00913 / \mathrm{K}$ & 241 -SX INSIALL 106-SX VAPOR PROBE \\
\hline $2 W-94-00914 / K$ & $241-5 X$ INSTALL SHMS IN SX FARM \\
\hline $2 \mathrm{~W}-94-00915 / \mathrm{K}$ & 241-SX RISER INSPECTION FOR S AND SX SHMS \\
\hline $2 \mathrm{~W}-94-00920 / \mathrm{K}$ & 241-SX INSIALL 109-SX SHMS TO VENT HEADER \\
\hline $2 W-94-00945 / K$ & 241-S INSTALL 102-S VAPOR PROBE \\
\hline $2 W-94-00946 / K$ & 241-S INSTALL SHMS IN S FARM \\
\hline $2 \mathrm{~W}-94-00947 / \mathrm{K}$ & 241-S INSTALL 111-S VAPOR PROBE \\
\hline $2 \mathrm{~W}-94-00949 / \mathrm{K}$ & 241-S INSTALL 112-S VAPOR PROBE \\
\hline $2 W-94-00988 / K$ & 241-T INSTALL 110-T VAPOR PROBE \\
\hline $2 W-94-00989 / K$ & 241-I INSTALL SHMS IN I FARM \\
\hline $2 W-94-00990 / K$ & 241-U INSTALL 103-U VAPOR PROBE \\
\hline $2 \mathrm{~W}-94-00991 / \mathrm{K}$ & $241-U$ INSTALL SHMS IN U FARM \\
\hline $2 \mathrm{~W}-94-00992 / \mathrm{K}$ & 241-U INSTALL 105-U VAPOR PROBE \\
\hline $2 \mathrm{~W}-94-00993 / \mathrm{K}$ & 241-U RISER INSPECTION FOR U AND T FARMS \\
\hline $2 \mathrm{~W}-94-00994 / \mathrm{K}$ & 241-U INSTALL 107-U VAPOR PROBE \\
\hline $2 \mathrm{~W}-94-00996 / \mathrm{K}$ & 241-U INSTALL 108-U VAPOR PROBE \\
\hline $2 \mathrm{~W} \cdot 94-00998 / \mathrm{K}$ & 241-U INSTALL 109-U VAPOR PROBE \\
\hline $2 E-95-00519 / K$ & INSTALL SHMS/AMMONIA MONITOR "AN" EXHAUSTERS \\
\hline $2 E-95-00604 / K$ & INSTALL SHMS/AMMONIA MONITOR AW-FARM EXHAUSTERS \\
\hline $2 E-95-00997 / K$ & PERFORM EXCAVATIONS, INSTALL CONDUIT FOR SHMS \\
\hline $2 E-95-01106 / K$ & PERFORM EXCAVATIONS, INSTALL CONDUIT FOR SHMS \\
\hline UNKNOWN, WORK PACKAGES HAVE BEEN & INSTALL 101 AND 103 SY SHMS \\
\hline
\end{tabular}

